\title{
Determining the contribution of NPM1 heterozygosity to NPM-ALK-induced lymphomagenesis
}

\author{
Fiona KE MD Duff', C Elizabeth Hook², Reuben M Tooze ${ }^{3}$, Brian J Huntly ${ }^{4}$, Pier Paolo Pandolfi ${ }^{5}$ and Suzanne D Turner ${ }^{1}$
}

\begin{abstract}
Heterozygous expression of Nucleophosmin (NPM1) predisposes to hematological malignancies in the mouse and cooperates with Myc in lymphomagenesis. NPM1 is therefore regarded as a haploinsufficient tumor suppressor. Heterozygous loss of NPM1 occurs as a result of the $t(2 ; 5)$, which generates the oncogenic fusion tyrosine kinase, NPM-anaplastic lymphoma kinase (ALK), a molecule underlying the pathogenesis of anaplastic large cell lymphoma (ALCL). Given the aforementioned role of NPM1 as a tumor suppressor, we hypothesized that NPM1 heterozygosity would cooperate with NPM-ALK in lymphomagenesis. In the event, we observed no difference in tumor latency, incidence or phenotype in NPM-ALK-transgenic mice heterozygous for NPM1 relative to transgenic mice expressing both NPM1 alleles. We propose that although the $t(2 ; 5)$ simultaneously reduces NPM1 allelic dosage and creates the NPM-ALK fusion protein, the two events do not cooperate in the pathogenesis of $A L C L$ in our mouse model. These data indicate that a tumor-suppressive role for NPM1 may depend on cellular and/or genetic context.
\end{abstract}

Laboratory Investigation (2011) 91, 1298-1303; doi:10.1038/labinvest.2011.96; published online 27 June 2011

KEYWORDS: ALCL; lymphoma; mouse model; NPM-ALK; NPM1

Nucleophosmin (NPM)-1 is a ubiquitously expressed, multifunctional phosphoprotein attributed with both oncogenic and tumor-suppressive functions. The protein has a key role in several cellular processes related to the control of cell growth and proliferation, and is haploinsufficient in the maintenance of genomic stability, as well as being a haploinsufficient suppressor of hematological malignancies in the mouse. ${ }^{1,2}$ The $t(2 ; 5)$, generating the fusion tyrosine kinase NPM-anaplastic lymphoma kinase (ALK), is considered the driving event in greater than $80 \%$ of $\mathrm{ALK}^{+}$ anaplastic large cell lymphoma (ALCL) cases. ${ }^{3}$ However, although the role of NPM-ALK in cellular proliferation and tumorigenesis has been well documented, these studies have not considered a potential role for NPM1 heterozygosity, occurring as a consequence of the $t(2 ; 5)$.

NPM1 heterozygosity accelerates disease onset in lymphoma-predisposed $E \mu-M y c$ mice. $^{2}$ We therefore reasoned that NPM1 heterozygosity may similarly cooperate with NPM-ALK in a transgenic mouse model of NPM-ALKinduced lymphomagenesis. However, no cooperation between these two events was observed, thus providing evidence to dismiss a causal role for NPM1 heterozygosity in the genesis of NPM-ALK ${ }^{+}$ALCL in this model system.

\section{MATERIALS AND METHODS Mice}

The generation of CD2/NPM-ALK transgenic mice was described previously as was the generation of NPM1 heterozygous mice. ${ }^{2,4}$ All mice were maintained on a C57BL/ 6J background (Jackson Laboratory, Charles River, Margate, UK). Mice were housed under specific pathogen free conditions in a barrier facility at the University of Cambridge, UK.

\section{Histology and Immunohistochemistry}

Mice displaying clinical signs were euthanized and postmortem performed examining all tissues. Tissue sections were stained with hematoxylin and eosin for histological examination. Immunohistochemical analysis was performed with the following antibody: ALK (Invitrogen, Paisley, UK).

\footnotetext{
'Division of Molecular Histopathology, Department of Pathology, University of Cambridge, Addenbrooke's Hospital, Cambridge, UK; ${ }^{2}$ Department of Histopathology, Addenbrooke's Hospital, Cambridge, UK; ${ }^{3}$ Leeds Institute of Molecular Medicine, University of Leeds, Leeds, UK; ${ }^{4}$ Department of Haematology, University of Cambridge, Cambridge, UK; and ${ }^{5}$ Cancer Genetics Program, Beth Israel Deaconess Cancer Center, Department of Medicine and Pathology, Beth Israel Deaconess Medical Center, Harvard Medical School, Boston, MA, USA

Correspondence: Dr SD Turner, PhD, Division of Molecular Histopathology, Department of Pathology, University of Cambridge, Lab Block Level 3, Box 231, Addenbrooke's Hospital, Hills Road, Cambridge CB20QQ, UK.

E-mail: sdt36@cam.ac.uk
}

Received 3 February 2011; revised 17 March 2011; accepted 19 April 2011 


\section{Western Blot}

Analysis of protein expression by western blot was performed as described previously ${ }^{5}$ using antibodies specific to NPM1 (NA24) (Abcam, Cambridge, UK) and actin (Sigma). Densitometric analysis of protein expression levels as a factor of the respective actin-loading control was performed using AIDA software and a Fuji LAS 4000 chemilluminescence analyzer (Raytek, Sheffield, UK).

\section{Statistics}

Kaplan-Meier survival curves were produced using MedCalc software (Mariakerke, Belgium).

\section{RESULTS}

To examine the impact of NPM1 genetic heterozygosity on the incidence, latency and pathological features of NPMALK-induced tumorigenesis, CD2/NPM-ALK ${ }^{+}$mice were crossed with $N P M 1^{+/-}$mice and the offspring (34 CD2/ NPM-ALK ${ }^{+} \mathrm{NPM1}^{+/-}$and $25 \mathrm{CD} 2 / \mathrm{NPM}-A L K^{+} \mathrm{NPM1}^{+/+}$ mice), as well as 39 genetically wild-type mice (littermates obtained from maintenance of the CD2/NPM-ALK mouse colony) and 12 NPM1 heterozygous mice, were monitored over a 2-year period (Figure 1 and Table 1). Of the 25 CD2/ NPM-ALK ${ }^{+} \mathrm{NPM1} 1^{+/+}$and $34 \mathrm{CD} 2 / \mathrm{NPM}-\mathrm{ALK} \mathrm{K}^{+} \mathrm{NPM1} 1^{+/-}$ mice under observation, $11(44 \%)$ and $13(38 \%)$ were
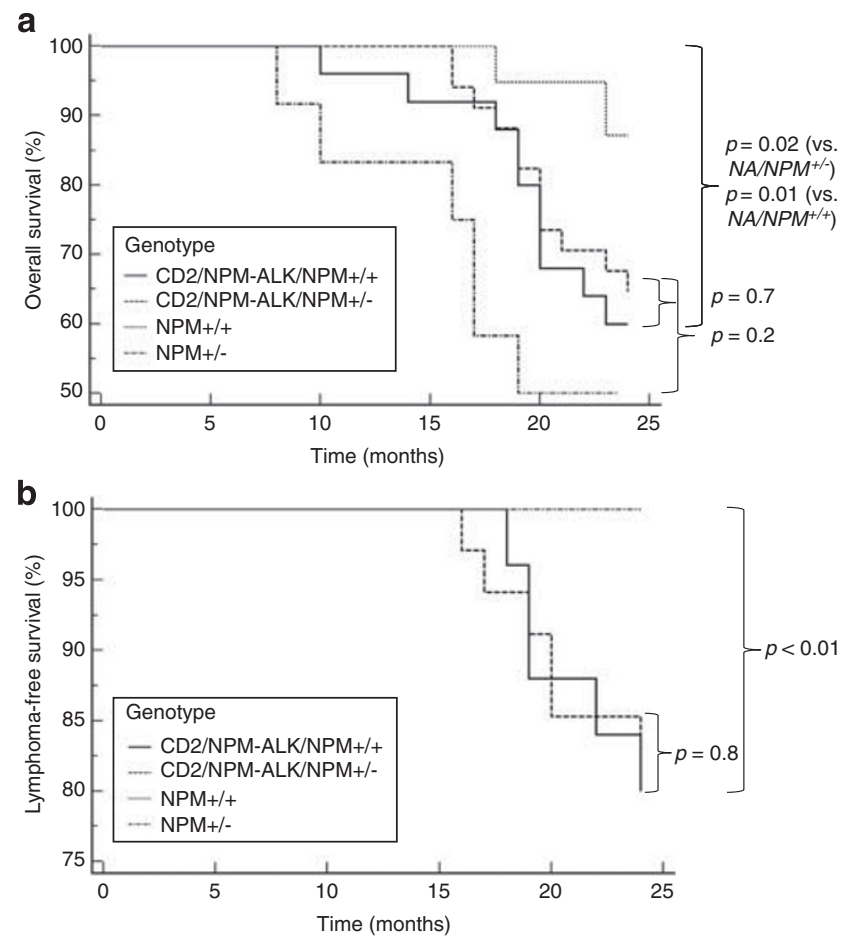

Figure 1 Survival and lymphoma incidence of CD2/NPM-ALK ${ }^{+} N P M 1^{+/+}$ and $C D 2 / N P M-A L K^{+} N P M 1^{+/-}$mice. Kaplan-Meier analysis of survival (a) and lymphoma incidence (b). $P$-values indicate the significance of survival or lymphoma incidence between two populations (indicated by brackets) and were assessed using the log-rank test. $P<0.05$ is considered to be significant. euthanized within the 2-year period, respectively (Figure 1a and Table 1). Lymphoma developed in both populations of NPM-ALK-expressing mice after a long latency that ranged from 10 to 24 months (Table 1). During this time, five (13\%) transgene-negative wild-type mice displayed clinical signs and were culled. There was no evidence of tumors and the cause of ill-health remains unknown but is highly likely to be age-related. Although CD2/NPM-ALK ${ }^{+} N P M 1^{+/+}$ and CD2/NPM-ALK ${ }^{+} N P M 1^{+/-}$mice succumbed to disease significantly earlier than wild-type mice (Figure 1a; $C D 2 /$ $N P M-A L K^{+} N P M 1^{+/+}$versus wild-type mice, $P=0.01 ; C D 2 /$ $\mathrm{NPM}-\mathrm{ALK}^{+} \mathrm{NPM1}^{+/-}$versus wild-type mice, $P=0.02$ ), the survival distribution of CD2/NPM-ALK transgenic mice expressing one or both alleles of NPM was equal (Figure 1a; $C D 2 / N P M-A L K^{+} N P M 1^{+/+}$versus $C D 2 / N P M-A L K^{+}$ $N P M 1^{+/-}$mice, $\left.P=0.7\right)$. Thus, heterozygous disruption of the NPM1 locus does not significantly accelerate disease onset or increase incidence in the context of oncogenic NPM-ALK signaling.

Table 1 details the macroscopic and microscopic pathology for each mouse that was culled on presentation of clinical signs or died unexpectedly ( $\leq 2$ years of age). At necropsy, many of the mice that died prematurely presented with enlarged lymph nodes with or without splenomegaly. In tissue sampled from mice of either genotype, histological analysis revealed the presence of extensive infiltrates characterized by atypical lymphocytes in spleen and lymph nodes that disrupted the normal tissue architecture (Figure 2a). Immunohistochemical analysis with an anti-ALK antibody confirmed expression of NPM-ALK in the tumor tissue (Figure 2b). Furthermore, western blot analysis revealed that loss of heterozygosity for NPM1 was not a feature of these tumors as NPM1 expression could be detected in tumors arising from both NPM1 heterozygous and NPM1 wild-type mice (Figure 2c). Amplification of NPM1 expression was likewise not a feature of the tumors that arose in the CD2/NPM-ALK ${ }^{+} N P M 1^{+/-}$mice, as protein lysates prepared from these tumors contained on average lower levels of NPM1 than tumor lysates extracted from $\mathrm{CD} 2 / \mathrm{NPM}-\mathrm{ALK}^{+} \mathrm{NPM1}{ }^{+/+}$mice (average densitometry level $=0.32$ compared with 2.24; Figure $2 \mathrm{c}$ ). B-cell lymphoma was the predominant tumor type presented by the CD2/ $N P M-A L K^{+} N P M 1^{+/+}$mice, occurring in five $(20 \%)$ mice, and was the only tumor type that developed in the CD2/NPM-ALK ${ }^{+} \mathrm{NPM1}^{+/-}$mice, occurring in six (18\%) of the mice (Table 1 and Figure 1b). Although low, the incidence of lymphoma was significantly different between the WT and the CD2/NPM-ALK ${ }^{+} N P M 1^{+/-}$cohorts $(P<0.01)$ but not between the CD2/NPM-ALK ${ }^{+} N P M 1^{+/-}$ and the $C D 2 / N P M-A L K^{+} N P M 1^{+/+}$groups $(P=0.8$; Figure 1b). A predominance to develop B-cell lymphomas is consistent with the tumor phenotype previously described for the CD2/NPM-ALK ${ }^{+} N P M 1^{+/+}$transgenic mice, although an incidence of $20 \%$ is lower than published observations. ${ }^{4}$ 


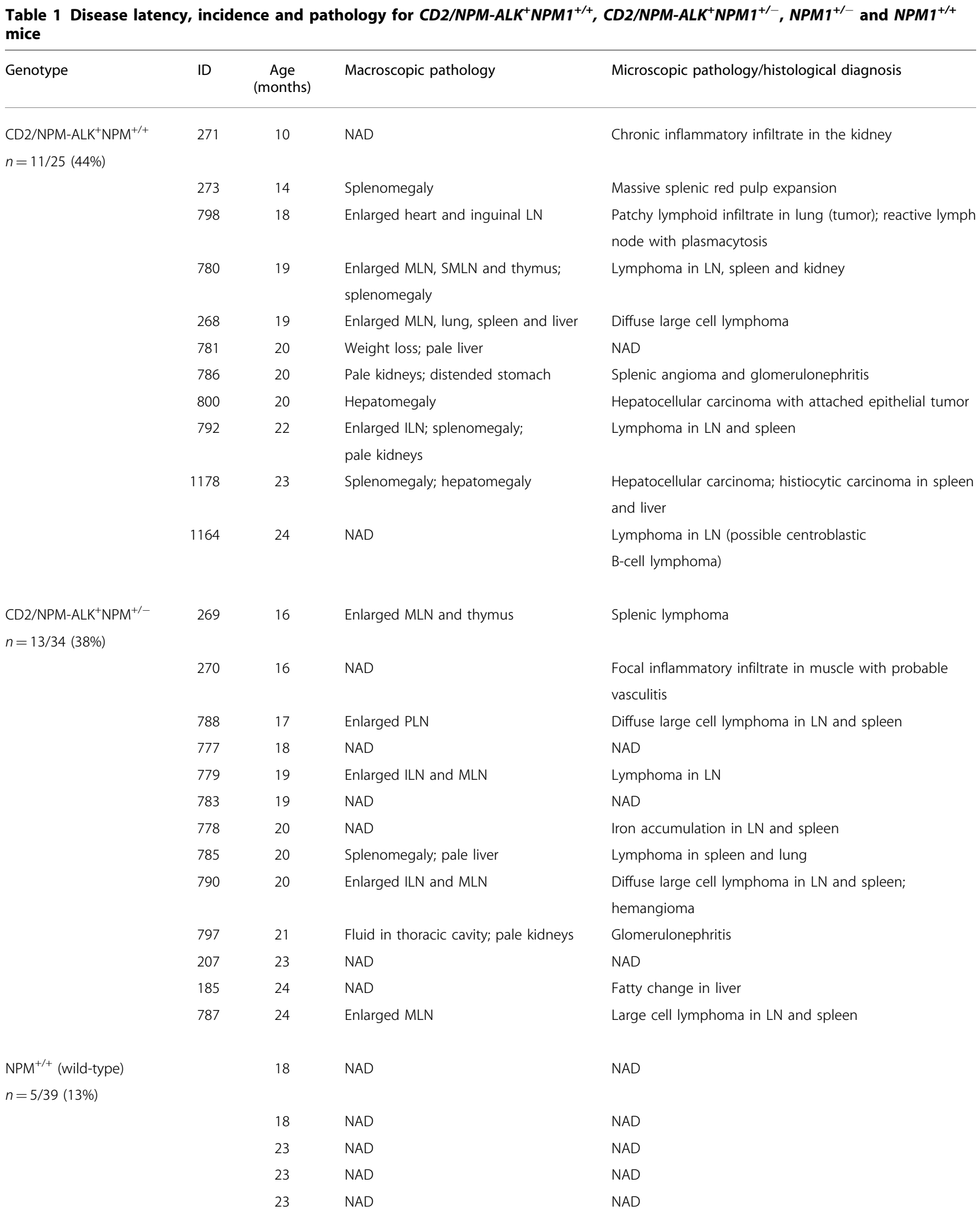


Table 1 Continued

\begin{tabular}{lccll}
\hline Genotype & ID & $\begin{array}{c}\text { Age } \\
\text { (months) }\end{array}$ & Macroscopic pathology & Microscopic pathology/histological diagnosis \\
\hline $\mathrm{NPM}^{+/-}$ & 37 & 16 & Pale kidney; enlarged ILN & NAD \\
$n=6 / 12(50 \%)$ & 17 & Cachexia & NAD \\
& 33 & 17 & Sore on neck & Low-grade hepatoma; glomerulonephritis \\
& 24 & 19 & Cachexia; hepatomegaly & Lymphadenitis with neutrophils \\
& 82 & Abcess in groin & NAD \\
\hline
\end{tabular}

$\mathrm{NAD}=$ no abnormality detected; $\mathrm{LN}=$ lymph node; $\mathrm{MLN}=$ mesenteric lymph node; ILN = inguinal lymph node; $\mathrm{SMLN}=$ sub-mandibular lymph node; $\mathrm{BM}=$ bone marrow.

$n$ relates to the number of mice presenting with an abnormal pathology among the cohort of mice of the specified genotype.

Other tumor types detected in the CD2/NPM$A L K^{+} \mathrm{NPM}^{+/+}$mice were largely non-hematological and included two cases of hepatocellular carcinoma (Table 1). However, one mouse (no. 1178) in addition to developing hepatocellular carcinoma presented with histiocytic sarcoma in the liver and spleen. Among both CD2/NPM$A L K^{+} \mathrm{NPM1}^{+/+}$and CD2/NPM-ALK ${ }^{+} \mathrm{NPM1}^{+/-}$cohorts, a small number of mice presented with an abnormal pathology in non-lymphocytic cells that was not associated with tumor formation, and therefore presumably unrelated to NPM-ALK expression, and possibly age-related (Table 1 and Figure 1a). These abnormal pathologies included inflammatory infiltrate in the heart and glomeruli of the kidney, as well as extensive red pulp expansion of the spleen. Finally, one $C D 2 / N P M-A L K^{+} N P M 1^{+/+}$and three $C D 2 /$ $N P M-A L K^{+} N P M 1^{+/-}$mice could not be associated with any distinct pathology following histological examination and therefore the reason for ill-health remains unknown (Table 1). Importantly, of those tumors for which NPM-ALK expression could be detected no significant difference in the incidence was observed between the two populations of mice (Figure $1 \mathrm{~b}$ ), indicating that NPM1 heterozygosity does not cooperate with NPM-ALK in lymphomagenesis or antagonize its oncogenic function in this model.

Although the CD2/NPM-ALK transgenic mice are prone to developing lymphoid malignancies, ${ }^{4}$ the $N P M 1^{+/-}$ genotype is reported to chiefly predispose to myeloid malignancies (either myeloproliferative disease-like myeloid leukemia (MPD-like ML) or ML). ${ }^{2}$ MPD-like $\mathrm{ML}$ in these mice is characterized by leukocytosis together with a vast increase in the number of well-differentiated myeloid cells in the bone marrow, spleen and non-hematopoietic tissues. ${ }^{2}$ ML is characterized by severe anemia, mild leukocytosis and thrombocytopenia, as well as the presence of leukemic blasts in affected tissues. ${ }^{2}$ Of the CD2/NPM$\mathrm{ALK}^{+} \mathrm{NPM1}^{+/+}$and CD2/NPM-ALK ${ }^{+} \mathrm{NPM1}^{+/-}$mice for which blood counts were taken, all mice exhibited perturbations in at least one of the following blood parameters: white blood cell, red blood cell or platelet count or hemoglobin levels (compared with the range obtained from the peripheral blood of 10-month-old wild-type litter mate mice $(n=10)$; Supplementary Table S1 and Supplementary Figure S1). However, the blood counts were not consistent with a diagnosis of MPD-like ML or ML. Extreme expansion of myeloid cells was not a feature of any of the tissues examined, which included bone marrow and analysis of blood smears (data not shown), and hence, no evidence of myeloid malignancy was observed in any of the mice, regardless of NPM1 genetic dosage. Equally, there was no evidence of myeloid disease in the $\mathrm{NPM1}^{+/-}$cohort (Supplementary Table S1). In none of the blood samples analyzed was there any trend to indicate a common deficiency or increase in blood cell count parameters in any of the cohorts, and group readings did not differ significantly from one another (Supplementary Figure S1). This disparity with the literature may reflect generation-specific differences, subtle changes in the genetic background of the mice following outcross to the $N P M-A L K$ transgenic lines or disparities in housing of the mice compared with the original mouse colonies.

\section{DISCUSSION}

Although transgene expression of NPM-ALK is sufficient to drive lymphoma development in several mouse models of NPM-ALK-induced tumorigenesis, none of these models faithfully recapitulate the human disease. ${ }^{4-9}$ For instance, disease latency is in most cases long and manifests after many months, suggesting the need for additional cooperating events to accelerate disease. In addition, in these and other models of NPM-ALK-driven lymphomagenesis, NPM-ALKexpressing B-cell lymphomas frequently develop despite supposed T-cell-restricted expression of the transgene. ${ }^{4,6}$ In all of these models, a potential role for $\mathrm{t}(2 ; 5)$-associated NPM1 allelic loss in tumor development has not been 
a
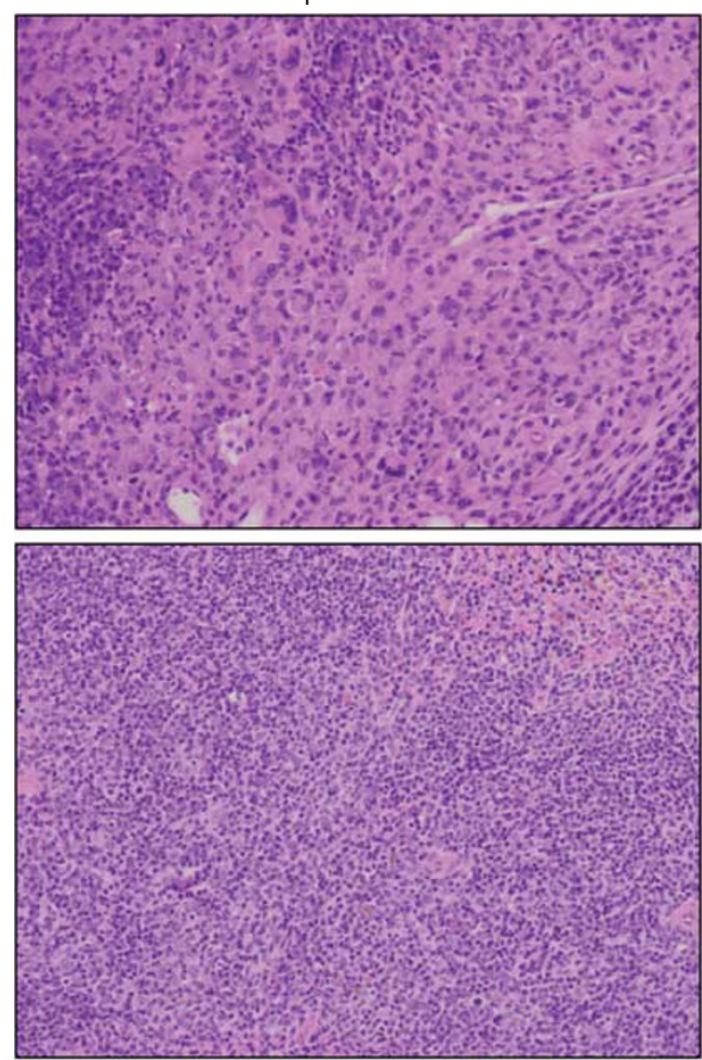

b

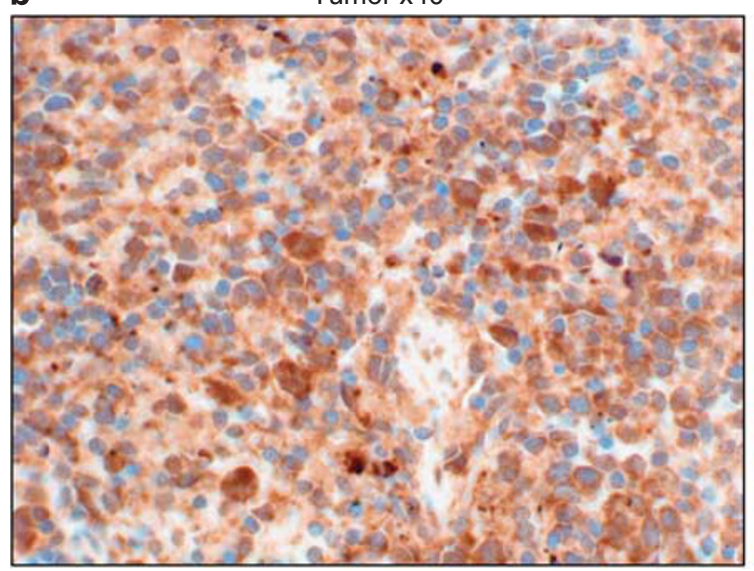

c

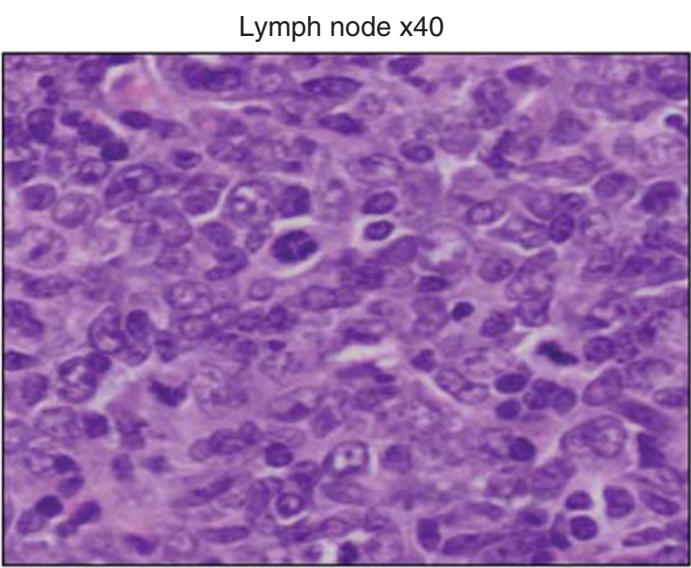

NPM-ALK ${ }^{+} \mathrm{NPM}^{+/+}$

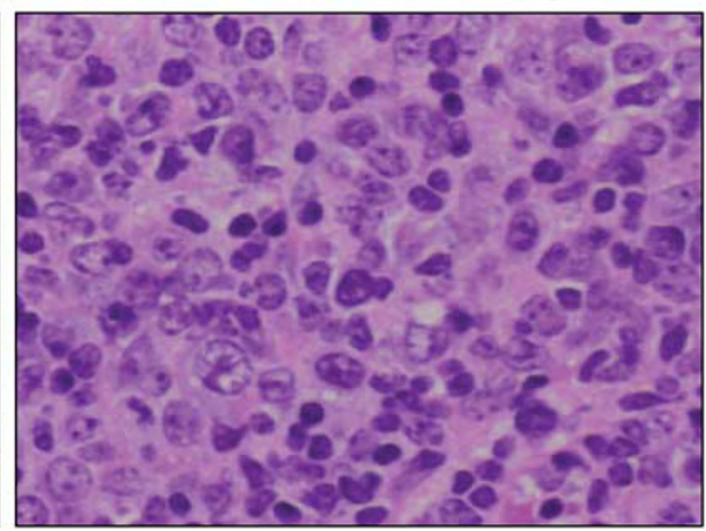

$\mathrm{NPM}-\mathrm{ALK}^{+} \mathrm{NPM}^{+/-}$
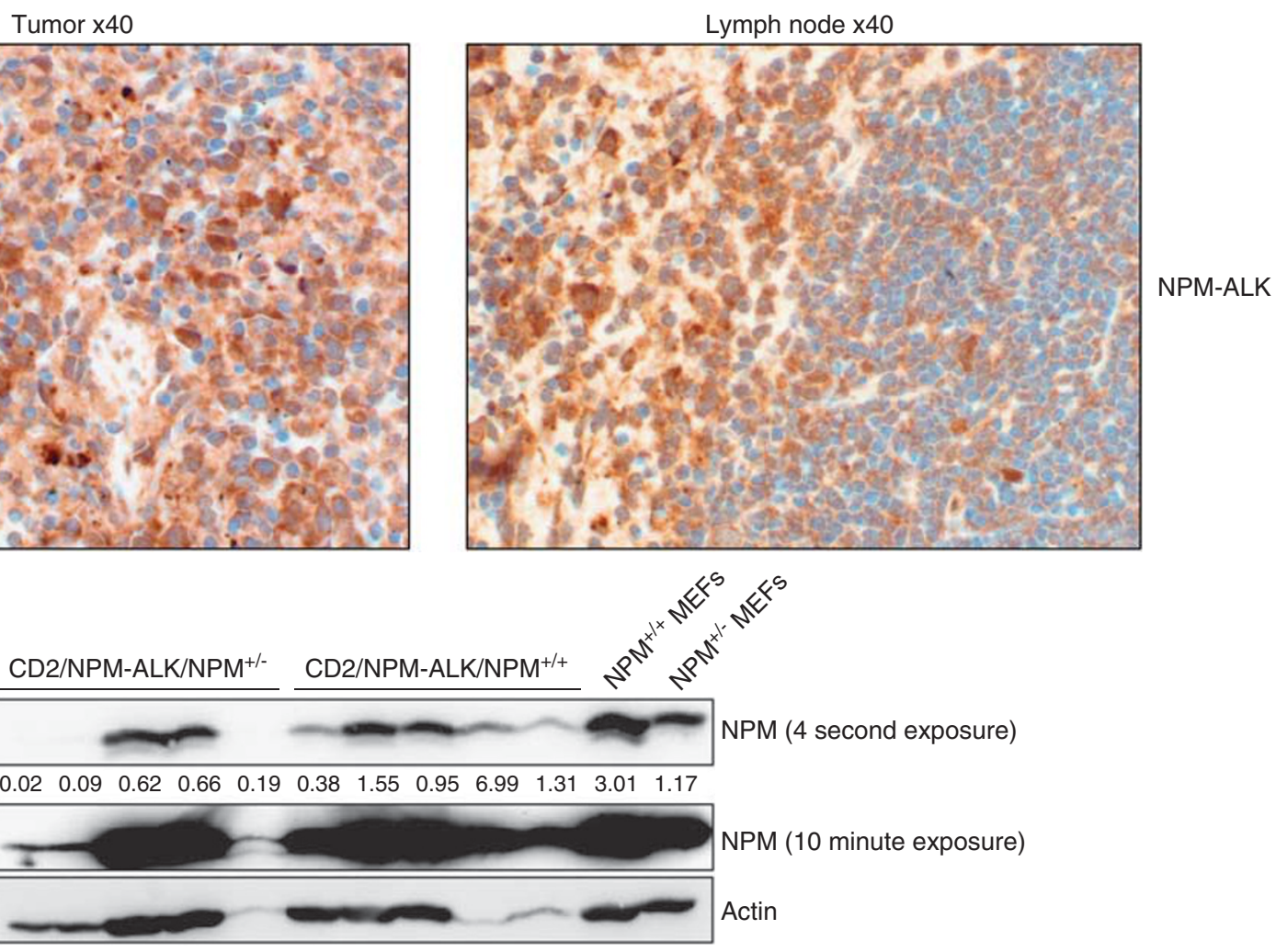
Figure 2 Histopathological presentation of tumors. (a) Hematoxylin and eosin-stained spleen and lymph node sections from $C D 2 / N P M-A L K^{+} N P M 1^{+/+}$ and $C D 2 / N P M-A L K^{+} N P M 1^{+/-}$mice presenting with tumor masses showing atypical lymphoid infiltrate disrupting the normal tissue architecture. (b) Immunohistochemical analysis of tumor sections to detect NPM-ALK expression. (c) Western blot analysis of NPM and actin expression levels in tumors isolated from $C D 2 / N P M-A L K^{+} N P M 1^{+/+}$and $C D 2 / N P M-A L K^{+} N P M 1^{+/-}$mice compared with $N P M^{+/-}$and $N P M^{+/+}$MEFs. The numbers refer to densitometric analysis of NPM expression levels in comparison to the respective actin control.

considered. Here, we addressed this issue by crossing mice expressing NPM-ALK from the CD2 promoter with NPM1 heterozygous mice and examining the resultant offspring. This mouse model was chosen because of the long latency to disease development and a lower disease penetrance, and hence, to provide a clear window in which our hypothesized increased disease incidence and shorter latency owing to a NPM1 heterozygous background could be observed. ${ }^{4}$ In the event, no difference in tumor latency, incidence or phenotype was observed, providing evidence that heterozygous expression of NPM1 does not cooperate with NPMALK in tumorigenesis in this model system. These results differ to the published observations that oncogenic Myc expression cooperates with NPM1 heterozygosity to accelerate tumor onset in lymphoma-predisposed mice. ${ }^{2}$ We propose that whereas Myc and NPM1 heterozygous expression have complementary functions in oncogenesis that permit cooperation, this is not the case for NPM-ALK expression and NPM1 heterozygosity although this remains to be determined by complementary in vitro studies. These findings are in accordance with analyses of $\mathrm{ALK}^{+}$ALCL patient data, indicating that tumors harboring $A L K$ fusions where the partner gene is not NPM1 exhibit an immunophenotypic, clinical and prognostic behavior that is indistinguishable from that of classical t $(2 ; 5)$ ALCL tumors, ${ }^{10}$ although the $t(2 ; 5)$ is the most common translocation, observed in greater than $80 \%$ of cases raising the question as to why this particular event is selected for. ${ }^{11}$ However, together, these data indicate that NPM1 heterozygosity does not contribute to the genesis of $t(2 ; 5)$-induced ALCL. It seems likely that the significance of this translocation lies largely, if not entirely, in the creation of an oncogenic tyrosine kinase that drives tumorigenesis. However, before this statement can be firmly concluded, it is necessary to examine the consequences of NPM1 heterozygosity in an $N P M-A L K$ transgenic model with a more penetrant disease phenotype or even a mouse in which NPM-ALK expression and NPM1 heterozygosity are achieved simultaneously, for example, using the translocator system developed by Rabbitts et al. ${ }^{12}$ Yet, one must consider that the phenotype observed in mouse models of cancer is influenced by a number of tangible and intangible variables and as such a direct correlation between mouse and human pathologies is often difficult to make, although we have observed that human NPM-
ALK forms some of the same hetero-complexes and occupies the same cellular sub-compartments in murine cells as has been observed in human cells (Supplementary Figure S2), suggesting that at least at the molecular level, NPM-ALK biology is equivalent in both mouse and human.

Supplementary Information accompanies the paper on the Laboratory Investigation website (http://www.laboratoryinvestigation.org)

\section{ACKNOWLEDGEMENTS}

This work was supported by Cancer Research UK (C19666 to SDT) and the Wellcome Trust (studentship to FKEM). SDT is a Leukaemia and Lymphoma Research Bennett Fellow (07006 to SDT), RT is a Cancer Research UK Senior Clinical Fellow and BJH is a Medical Research Council Senior Clinical Research Fellow.

\section{DISCLOSURE/CONFLICTS OF INTEREST}

The authors, SDT, FKEM, CEH, BJH and RT have no conflicts of interest to disclose.

1. Sportoletti P, Grisendi S, Majid SM, et al. Npm1 is a haploinsufficient suppressor of myeloid and lymphoid malignancies in the mouse. Blood 2008;111:3859-3862.

2. Grisendi $S$, Bernardi R, Rossi $M$, et al. Role of nucleophosmin in embryonic development and tumorigenesis. Nature 2005;437: 147-153.

3. Benharroch D, Meguerian-Bedoyan Z, Lamant L, et al. ALK-positive lymphoma: a single disease with a broad spectrum of morphology. Blood 1998;91:2076-2084.

4. Turner SD, Merz H, Yeung $D$, et al. CD2 promoter regulated nucleophosmin-anaplastic lymphoma kinase in transgenic mice causes B lymphoid malignancy. Anticancer Res 2006;26:3275-3279.

5. Turner SD, Tooze $\mathrm{R}$, Maclennan $\mathrm{K}$, et al. Vav-promoter regulated oncogenic fusion protein NPM-ALK in transgenic mice causes B-cell lymphomas with hyperactive Jun kinase. Oncogene 2003;22: 7750-7761.

6. Chiarle R, Gong JZ, Guasparri I, et al. NPM-ALK transgenic mice spontaneously develop T-cell lymphomas and plasma cell tumors. Blood 2003;101:1919-1927.

7. Turner SD, Alexander DR. What have we learnt from mouse models of NPM-ALK-induced lymphomagenesis? Leukemia 2005;19:1128-1134.

8. Kuefer MU, Look AT, Pulford K, et al. Retrovirus-mediated gene transfer of NPM-ALK causes lymphoid malignancy in mice. Blood 1997;90: 2901-2910.

9. Jager R, Hahne J, Jacob A, et al. Mice transgenic for NPM-ALK develop non-Hodgkin lymphomas. Anticancer Res 2005;25:3191-3196.

10. Falini B, Pileri S, Zinzani PL, et al. ALK+lymphoma: clinico-pathological findings and outcome. Blood 1999;93:2697-2706.

11. Swerdlow SH. WHO Classification of Tumors of Haematopoietic and Lymphoid Tissues. International Agency for Research on Cancer: Lyon, 2008.

12. Rabbitts TH, Appert A, Chung G, et al. Mouse models of human chromosomal translocations and approaches to cancer therapy. Blood Cells Mol Dis 2001;27:249-259. 